\title{
The Frequency of Ankylosing Spondylitis Among Patients With Familial Mediterranean Fever and the Relationship With M694V Mutation
}

\author{
Zühal ÖRNEK, ${ }^{1}$ Nesibe KARAHAN YEŞİL, ${ }^{2}$ Metin IŞIK, ${ }^{3}$ Hatice IŞIK ${ }^{4}$ \\ ${ }^{1}$ Department of Pediatrics, Medical Faculty of Bülent Ecevit Universiy, Zonguldak, Turkey \\ ${ }^{2}$ Department of Internal Medicine, Division of Rheumatology, Ankara Training and Research Hospital, Ankara, Turkey \\ ${ }^{3}$ Department of Internal Medicine, Division of Rheumatology, Medical Faculty of Bülent Ecevit Universiy, Zonguldak, Turkey \\ ${ }^{4}$ Department of Obstetrics and Gynecology, Medical Faculty of Bülent Ecevit Universiy, Zonguldak, Turkey
}

As an auto-inflammatory disease, familial Mediterranean fever (FMF) is characterized by recurrent attacks of fever and serositis. The second most frequent manifestation of the disease is arthritis which mostly presents as a monoarthritis of the hip or knee. ${ }^{1}$ Furthermore, the frequency of sacroiliitis is also increased among patients with FMF and some authors blame M694V mutation for this relationship. ${ }^{2}$ In this article, we present our experience in the coexistence of FMF and ankylosing spondylitis (AS) and the M694V mutation ratio among these patients.

Our medical database included 474 patients with AS and 111 patients with FMF. Of these patients, 19 (10 males and 9 females) were diagnosed with both, meaning that $4 \%$ of the patients with AS had FMF and $17.1 \%$ of the patients with FMF had AS. A written informed consent was obtained from each patient. The median age at diagnosis for FMF was 27 (range 13 to 40 ) and 36 (range 15 to 42 ) for AS. Totally, 17 patients (89.5\%) had M694V mutation and nine (47.4\%) were homozygotes. Eighteen patients were tested for human leukocyte
antigen-B27 and six had positive results (33.3\%). All were taking daily colchicine and 12 (63.1\%) were on sulfasalazine therapy. There were six patients (31.5\%) taking anti-tumor necrosis alpha agents (four taking adalimumab, one taking etanercept, and one taking golimumab) and the last patient was taking indomethacin. There were two patients (10.5\%) with pathologically proven amyloidosis. Totally, nine patients $(47.4 \%)$ had peripheral and 10 (52.6\%) had axial disease.

In conclusion, AS is very common among patients with FMF and nearly one third of these patients need anti-tumor necrosis alpha agents for treatment. Therefore, all patients with FMF should be questioned for inflammatory back pain.

\section{Declaration of conflicting interests}

The authors declared no conflicts of interest with respect to the authorship and/or publication of this article.

\section{Funding}

The authors received no financial support for the research and/or authorship of this article. 


\section{REFERENCES}

1. Tunca M, Akar S, Onen F, Ozdogan H, Kasapcopur $\mathrm{O}$, Yalcinkaya F, et al. Familial Mediterranean fever (FMF) in Turkey: results of a nationwide multicenter study. Medicine (Baltimore) 2005;84:111.

2. Akkoc N, Gul A. Familial Mediterranean fever and seronegative arthritis. Curr Rheumatol Rep 2011;13:388-94. 